\title{
ON THE RADIAL DEPENDENCE OF ISOVECTOR \\ MESON EXCHANGE CURRENTS
}

\author{
J.-F. Mathiot* \\ CERN - Geneva
}

\begin{abstract}
The structure of isovector meson exchange currents for the electrodisintegration of the deuteron near threshold is studied as a function of the relative distance between the two nucleons. Different forms for the operators (with and without phenomenological form factors or model independent derivation from the Paris NN potential) are studied for $\pi$ - and $\rho$-exchange contributions and for different momentum transfers. Some consequences concerning the elementary degrees of freedom in the deuteron are discussed.
\end{abstract}

* On leave from Division de Physique Théorique ${ }^{+}$, Institut de Physique Nucléaire, F-91406 Orsay Cedex.

+ Laboratoire associé au C.N.R.S.

CERN-TH. 4576/86

October 1986 
The interest in Meson Exchange Current (MEC) contributions to isovector magnetic transitions has already been recognized a long time ago. In particular two very simple processes have been shown essential in understanding such two-body currents. At zero momentum transfer, the thermal np capture cross-section is given to $90 \%$ by the impulse approximation contribution [1]:

$$
\begin{aligned}
& \sigma_{\exp }=334.2 \pm 0.5 \mathrm{mb}, \\
& \sigma_{\mathrm{IA}}=302.5 \pm 4 \mathrm{mb},
\end{aligned}
$$

the uncertainty in the theoretical value reflecting the uncertainty in the asymptotic behaviour of the nuclear wave function. The remaining $10 \%$ was successfully attributed to MECs due to $\pi$ exchange [2]. This interpretation was confirmed later on with more complete calculations [3-5] (including in particular form factors and $\rho$-exchange contributions [5]). This however implies the use of a short-range meson-nucleon form factor, which should also enter the description of the NN potential used to calculate the two-body wave function.

This scheme proved also successful in explaining the deuteron electrodisintegration (DE) cross-section near threshold [6-11] at high momentum tranfer $\left(\mathrm{q}^{2} \approx 6-30 \mathrm{fm}^{-2}\right)$. In addition this process put very precise conditions on the range of the form factors and on the $\rho-\mathrm{N}$ tensor coupling constant .

The main issue of that description of the DE at high momentum transfer is precisely to investigate the short range behaviour of the nuclear wave function. Near threshold, the transition to the ${ }^{1} \mathrm{~S}_{0}$ scattering state dominates the cross section. However, the exact range of the MEC contributions at various momentum transfer is not at all apparent on the cross section. We shall study this particular point by looking at the elementary matrix elements of the two-body currents. This study extends some results already published $[12,13]$.

The dominant MECs for the purely isovector transition ${ }^{3} \mathrm{~S}_{1}+{ }^{3} \mathrm{D}_{1} \rightarrow{ }^{1} \mathrm{~S}_{0}$ are represented in figures 1.a (seagull diagram), 1.b (mesonic diagram); and 2 (isobar diagram). They are associated with $\pi$ and $\rho$ meson exchange. The last diagram does not give a very large 
correction, once the $\rho$ meson contribution has been taken into account. Furthermore, as is already well known, this contribution is totally transverse in the transferred momentum and therefore not constrained by current conservation. For clarity of the discussion we then disregard this contribution in the following. The first two diagrams are model-independent in the sense that they should obey the current conservation equation :

$$
\nabla . \mathrm{J}^{\mathrm{ex}}+\mathrm{i}[\mathrm{V}, \rho]=0,
$$

where $\mathrm{V}$ is the $\mathrm{NN}$ potential and $\rho$ the charge density operator. This equation is obviously verified for point particles, in leading $1 / \mathrm{M}$ order, with the contribution corresponding to figs. 1 and the NN potential induced by the exchange of $\pi$ and $\rho$ mesons.

However, when form factors are introduced at the meson-nucleon vertices, MECs have also to be modified in a non-trivial way in order still to verify eq.(1). With the usual parametrization of the form factor in momentum space:

$$
\mathrm{F}_{\mathrm{MNN}}\left(\mathrm{k}^{2}\right)=\frac{\Lambda_{\mathrm{M}}^{2}-\mathrm{m}_{\mathrm{M}}^{2}}{\Lambda_{\mathrm{M}}^{2}-\mathrm{k}^{2}} \quad \stackrel{\mathrm{k}_{\mathrm{o}}=0}{\rightarrow} \frac{\Lambda_{\mathrm{M}}^{2}-\mathrm{m}_{\mathrm{M}}^{2}}{\Lambda_{\mathrm{M}}^{2}+\mathrm{k}^{2}}
$$

such derivation of MEC is straightforward [11]. The values of the cut-off masses $\Lambda_{M}$ are of course to be related in some way to the cut-off used in the construction of the NN potential itself.

The derivation of MEC operators directly from a realistic NN potential (Paris potential in particular [14]) has recently been proposed [15]. This procedure, based on the current conservation equation (1), consists in reconstructing generalized $\pi$-and $\rho$-exchange contributions directly from the numerical parametrization of the potential. These contributions automatically include form factors consistent with the potential. Hovewer, the extraction of $\pi$ and $\rho$ channels in such derivation is not free from ambiguity, especially at very short distances, where other mesons of higher mass can be mixed up. We propose in this letter to see exactly how these two approaches compare with each other, given that the differential cross-section of the DE near threshold is almost identical in both cases. 
The differential cross-section is given, in the backward direction, by :

$$
\frac{d^{2} \sigma}{d \Omega d w} \approx \sigma_{0}\left|<n_{p}\|T\| d>\right|^{2}
$$

where $\sigma_{0}$ is a kinematical constant and the operator $\mathrm{T}$ is directly related to the electromagnetic current [11]. It can be separated into one- and two-body contributions,$T=T^{(1)}+T^{(2)}$, with

$$
\begin{aligned}
& \left\langle n_{p}\left\|T^{(1)}\right\| d\right\rangle=i \frac{q}{2 M} G_{M}^{r}\left(q^{2}\right) \frac{1}{\sqrt{2 \pi}} . \\
& \int_{0}^{\infty} d r\left[j_{0}\left(q r_{/ 2}\right) u(r)-j_{2}\left(q r_{2}\right) \frac{w(r)}{\sqrt{2}}\right] \mu_{0}(r),
\end{aligned}
$$

whereas $\mathrm{T}^{(2)}$ is the contribution coming from the two exchange diagrams shown in figs. 1.a and b. We have :

$$
\begin{aligned}
& \left\langle n p\left\|T_{a}^{(2)}\right\| d\right\rangle=i 2 \sqrt{\frac{2}{\pi}} F_{1}^{r}\left(q^{2}\right) \frac{g_{\pi N N}^{2}}{16 \pi M^{2}} \int_{0}^{\infty} d r \frac{e^{-m_{\pi^{n}}}}{r^{2}}\left(1+m_{\pi} r\right) . \\
& j_{1}\left(q r_{2}\right)\left[\mu(r)+\frac{w(r)}{\sqrt{2}}\right] \mu_{0}(r), \\
& \left.\operatorname{unp}\left\|T_{b}^{(2)}\right\| d\right\rangle=\frac{i}{3} \sqrt{\frac{2}{\pi}} F_{1}^{v}\left(q^{2}\right) \frac{g_{\pi N N}^{2}}{16 \pi M^{2}} \int_{0}^{\infty} d r\{ \\
& \mu(r) \mu_{0}(r)\left[\left(\frac{d^{2}}{d r^{2}}+\frac{2}{r} \frac{d}{d r}\right) t_{0}+\left(\frac{d^{2}}{d r^{2}}+\frac{5}{r} \frac{d}{d r}+\frac{3}{r^{2}}\right) t_{2}\right] \\
& \left.+\frac{w(r)}{\sqrt{2}} \mu_{0}(r)\left[\left(\frac{d^{2}}{d r^{2}}-\frac{1}{r} \frac{d}{d r}\right) t_{0}+\left(\frac{d^{2}}{d r^{2}}+\frac{2}{r} \frac{d}{d r}-\frac{6}{r^{2}}\right) t_{2}\right]\right\},
\end{aligned}
$$

with $t_{e}=\int_{0}^{1} \frac{e^{-\alpha r}}{\alpha} f_{1}\left(\beta q r_{2}\right) d \beta \quad$ and $\quad \alpha=\sqrt{m_{\pi}^{2}+\frac{q^{2}}{4}\left(1-\beta^{2}\right)}$

In these expressions $\mathrm{u}_{0}(\mathrm{r}), \mathrm{u}(\mathrm{r})$ and $\mathrm{w}(\mathrm{r})$ are respectively the ${ }^{1} \mathrm{~S}_{0},{ }^{3} \mathrm{~S}_{1}$ and ${ }^{3} \mathrm{D}_{1}$ wave functions. The isovector electromagnetic form factors are $G_{M}{ }^{V}\left(q^{2}\right)$ and $F_{1} v\left(q^{2}\right)$ [13]. The dominant part of the $\rho$-exchange contribution is of similar type, and can be easily calculated by exchanging $g_{\pi \mathrm{NN}}$ 
with $g_{\rho N N}(1+\kappa)$ and $m_{\pi}$ with $m_{\rho}$. Here $\kappa$ is the $\rho$-nucleon tensor coupling constant whose value is taken to be $k=6.6$ (strong coupling).

In order to investigate the dependence of these matrix elements on the relative distance between the two nucleons, we rewrite them in the following form :

$$
\langle n p\|T\| d\rangle=i \frac{q}{\sqrt{2 \pi}} \int_{0}^{\infty} d r \eta(r, q) \text {, }
$$

and we plot in figs. 3 to 8 the function $\eta(r, q)$ for three different values of the transferred momentum : $\mathrm{q}^{2}=0 \mathrm{fm}^{-2}$ (figs. 3,6 ), $\mathrm{q}^{2}=15 \mathrm{fm}^{-2}$ (figs. 4,7) and $\mathrm{q}^{2}=30 \mathrm{fm}^{-2}$ (figs. 5,8 ). The contribution of $\pi$ - and $\rho$-exchange is also shown separately. For comparison we show in fig. 9 the contribution coming from the impulse approximation amplitude for the same quantity .

The solid lines in figs. 3-5 and 6-8 represent $\eta(r, q)$ for bare meson exchange operators associated with $\pi$ - and $\rho$-exchange respectively. In that case nucleons and mesons are considered as point particles. The dashed lines correspond to MEC operators modified by hadronic form factors of the form (2), with the following cut-off parameters :

$$
\begin{aligned}
& \Lambda_{\pi}=1.25 \mathrm{Gev} / \mathrm{c} \\
& \Lambda_{\rho}=1.50 \mathrm{Gev} / \mathrm{c}
\end{aligned}
$$

The operators are derived in order still to verify the current conservation equation (1) with $\pi$ and $\rho$-exchange potential, and the Paris potential is used to calculate the two-body wave functions. Finally, the dash-dotted lines correspond to MEC operators derived directly from the Paris potential, according to refs. 15 .

We proceed now to discuss all these results in detail. We first have to note that the quantity $\eta(r, q)$ incorporates the two-body wave function of the initial and final states. This is of course important since we want to discuss the sensitivity of the differential cross-section to short-range phenomena.

The comparison of these different figures leads to interesting conclusions. First of all, the bare $\pi$-exchange contribution to $\eta(r, q)$ (solid line) is peaked as expected at distances of the order of $1.4 \mathrm{fm}$ for $\mathrm{q}^{2}=0 \mathrm{fm}^{-2}$, and at relatively smaller distances for higher momentum transfer. However, at $\mathrm{q}^{2}=30 \mathrm{fm}^{-2}$, the MECs contribute mostly for relative distances of the order 
of $1 \mathrm{fm}$. This has to be compared to the range of the attractive part of the NN potential, which is about $1.2(0.9) \mathrm{fm}$ for the ${ }^{3} \mathrm{~S}_{1}\left({ }^{1} \mathrm{~S}_{0}\right)$ channel (with the Paris potential [14]). This point is further confirmed when hadronic form factors of relatively short range are introduced (dashed lines). These form factors have been chosen in order to be consistent with the description of the Paris potential (cut-off at short distances [11]), and with the experimental cross-section of the $D E$. The functions $\eta(r, q)$ are slightly reduced at distances less than $1 \mathrm{fm}$, and the negative part of MEC at very short distances is enhanced. This part comes almost entirely from the contribution of the mesonic diagram (fig. 1.b). The overall reduction of the functions $\eta(r, q)$ by the form factors is small, even at high momentum transfer. The total (integrated) amplitude for these different cases is indicated in the table. The function $\eta(r, q)$ for the generalized $\pi$-exchange current [15] (dash-dotted line) is very similar in shape and amplitude to the phenomenological derivation of MEC (dashed line). The cut-off at short distances is only slightly weaker, i.e. the equivalent mass parameter in eq. 2 should be larger. We estimate this parameter at about 1.4 Gev/c to get exactly the same result with both approaches. This is confirmed by the integrated values reported in the table. From a phenomenological point of view, and if we accept the derivation of the generalized MEC for the very short distances, we can conclude that the effective range of the mass parameter to cut off $\pi$-nucleon vertices should be at least larger than $1.25 \mathrm{Gev} / \mathrm{c}$, according to the use of the Paris potential to calculate the nuclear wave function.

The results for $\rho$ meson contribution are also very interesting. The bare meson exchange amplitude $\eta(r, q)$ shown in figs. $6-8$ (solid line) is peaked at about $0.8-0.9 \mathrm{fm}$ for the whole range of momentum transfer. This is reasonable given the large mass of the $\rho$ meson. Furthermore its relative contribution to the $\pi$ meson (for bare operators) is approximately $25 \%$, $35 \%, 60 \%$ for $\mathrm{q}^{2}=0,15$ and $30 \mathrm{fm}^{-2}$ respectively. Once form factors of the type (2) are taken into account (dashed line), the $\rho$ meson contribution is reduced appreciably and its relative contribution to the $\pi$ meson is then $15 \%, 25 \%, 30 \%$ for the three different momentum transfers. However, such contribution is essential in getting the experimental cross-section at $\mathrm{q}^{2} \approx 25-30$ $\mathrm{fm}^{-2}$, since the impulse approximation and $\pi$-exchange contribution are exactly cancelling each other [11]. The overall shape of the contribution is still peaked at about $0.8-1 \mathrm{fm}$. This of course 
is a consequence of the repulsive part of the $\mathrm{NN}$ potential which pushes the two nucleons apart. One can easily see that, even at $\mathrm{q}^{2}=30 \mathrm{fm}^{-2}$, the $\rho$ meson contribution is still one third of the long-range $\pi$-exchange, so that it cannot be ignored in such a model based on the one-boson exchange picture of the NN potential.

Finally, figs. 6-8 (dash-dotted line) shows $\eta(r, q)$ for the generalized $\rho$ meson contribution. While the total contribution of MECs in that case is almost identical to that of the phenomenological derivation presented above (see the table), the shape and amplitude are somewhat different. The width of the contribution is larger and consequently the amplitude is reduced by about $15 \%$, the function $\eta(r, q)$ extending to larger distances. This however can easily be attributed to the finite width of the $\rho$ meson (or to the $2 \pi$ contribution to the $\mathrm{NN}$ potential). This width is consistently taken into account in the latter derivation while the $\rho$ meson is considered as a zero-width particle in the former. The relative contribution to $\pi$ meson exchange is almost the same in both approaches. Once the finite width correction is made, there should be neither qualitative nor quantitative differences between the two approaches. In particular the range of the form factor, eqs. 2 and 7, should be consistent with the Paris potential, together with the strong value for the $\rho$-nucleon tensor coupling constant $(\kappa=6.6)$ used in deriving the $\rho$ meson contribution.

We think that the various results discussed in this letter are important in the light of the recent developments concerning the influence of the nucleon internal structure in intermediate energy nuclear physics. The deuteron electrodisintegration near threshold is certainly one of the best examples where precise tests of the nuclear models can be performed. It turns out that the DE cross-section at high momentum transfer $\left(q^{2} \approx 15-30 \mathrm{fm}^{-2}\right)$ is sensitive to the exact form of the currents and wave functions, essentially because of the strong cancellation between the impulse approximation and $\pi$-exchange contributions. This however enables us to determine the different parameters of the nuclear models, and to find a consistent description of the cross section, with respect both to current conservation and to the description of the NN potential itself. We show in this letter that such a consistent description has almost nothing to do with the 
very short-range part neither of the wave function, nor of the NN potential $(r<0.8 \mathrm{fm}$ for the Paris potential). One could ask whether the situation should change at higher momentum transfer. The cross-section presents a large plateau after the first minimum $\left(\mathrm{q}^{2} \approx 35-50 \mathrm{fm}^{-2}\right)$ due essentially to the one-body contribution. This latter will then mask the MEC corrections and consequently would be less sensitive to the details of the electromagnetic operators, except for relativistic corrections to the one-body current and electromagnetic form factors. One then must certainly go beyond $\mathrm{q}^{2}=2(\mathrm{Gev} / \mathrm{c})^{2}$ (or $50 \mathrm{fm}^{-2}$ ) in order to investigate explicit signatures of the quark degrees of freedom in the deuteron wave function. This implies in particular that the structure of the NN potential at very short distances should be reinterpreted in terms of these new degrees of freedom and that electromagnetic one- and two-body operators should still verify the current conservation equation.

I would like to thank T.E.O.Ericson and S. Platchkov for useful discussions, comments and suggestions. I gratefully acknowledge a Fellowship from the C.E.R.N. theoretical division. 


\begin{tabular}{|l|l|lcl|llll|}
\hline $\begin{array}{l}\mathrm{q}^{2} \\
\left(\mathrm{fm}^{-2}\right)\end{array}$ & \multicolumn{9}{c}{$\begin{array}{c}\mathrm{MEC}(\pi) \\
\mathrm{b}\end{array}$} & $\mathrm{a}$ & $\mathrm{b}$ & $\mathrm{c}$ & $\mathrm{a}$ & $\mathrm{MEC}(\rho)$ & $\mathrm{b}$ & $\mathrm{c}$ \\
\hline 0 & 1.0 & $4.510^{-2}$ & $4.210^{-2}$ & $4.310^{-2}$ & $1.110^{-2}$ & $6.810^{-3}$ & $7.010^{-3}$ \\
15 & $-2.510^{-3}$ & $1.110^{-2}$ & $9.210^{-3}$ & $9.810^{-3}$ & $3.710^{-3}$ & $2.210^{-3}$ & $2.210^{-3}$ \\
30 & $-2.710^{-3}$ & $3.510^{-3}$ & $2.810^{-3}$ & $3.210^{-3}$ & $1.710^{-3}$ & $8.810^{-4}$ & $8.810^{-4}$ \\
\hline
\end{tabular}

Table: Integrated values of $\eta(r, q)$ over all space for different forms of the operators and different momentum transfers. a: bare operator; $b$ : with dipole form factor according to eq. 7 ; c: model-independent operators [15] derived from the Paris NN potential. All the quantities are relative to the impulse approximation value at zero momentum transfer. 


\section{References}

[1] H.P. Noyes, Nucl. Phys. 74 (1965) 508

A.E. Cox et al. , Nucl. Phys. 74 (1965) 497

[2] D. O.Riska and G.E. Brown, Phys. Lett. 38B (1972) 994

[3] M. Gari and A. Huffmann, Phys. Rev. C7 (1973) 994

[4] M. Colocci, B. Mosconi and P. Ricci , Phys. Lett. 45B (1973) 224

[5] J.-F. Mathiot, Phys. Lett. 115B (1982) 174

[6] J. Hockert et al , Nucl. Phys. A217 (1973) 14

[7] J.A. Lock and L.L. Foldy, Ann. of Phys. 93 (1975) 276

[8] W. Fabian and H. Arenhövel, Nucl. Phys. A258 (1976) 461

[9] B. Sommer, Nucl. Phys. A308 (1978) 263

[10] W. Leidemann and H. Arenhövel, Nucl. Phys. A393 (1983) 385

[11] J.F. Mathiot, Nucl. Phys. A412 (1984) 201

[12] J.-F. Mathiot, Chr. Bargholtz and G.E. Brown, Nucl. Phys. A397 (1983) 365

[13] J.-F. Mathiot, Nucl. Phys. A446 (1985) 123c

[14] M. Lacombe et al , Phys. Rev. C21 (1980) 861

[15] D. O. Riska, Phys. Scr. 31 (1985) 471

A. Buchmann, W. Leidemann and H. Arenhövel, Nucl. Phys. A443 (1985) 726 


\section{Figure captions}

Figure 1: Dominant isovector meson exêháng ć currents : a. seagull diagram; $\mathrm{b}$. mesonic diagram.

Figure 2: Contribution of the isobar excitation in first order.

Figure 3: Structure of MEC as a function of $r$, for $q^{2}=0 \cdot \mathrm{fm}^{-2}$. The MEC operators are associated with $\pi$-exchange without form factors (solid line), with form factors according to eqs. 2 and 7 (dashed line) or to generalized $\pi$-exchange of refs. 15 (dash-dotted line).

Figure 4: Same as fig. 3 but for $q^{2}=15 \mathrm{fm}^{-2}$.

Figure 5: Same as fig. 3 but for $\mathrm{q}^{2}=30 \mathrm{fm}^{-2}$.

Figure 6: Same as fig. 3 but for $\rho$ meson exchange.

Figure 7: Same as fig. 4 but for $\rho$ meson exchange.'

Figure 8: Same as fig. 5 but for $\rho$ meson exchange.

Figure 9: Structure of the impulse approximation contribution as a function of $r$, for different momentum transfers: $q^{2}=0 \mathrm{fm}^{-2}$ (solid line), $\mathrm{q}^{2}=15 \mathrm{fm}^{-2}$ (dashed line) and $\mathrm{q}^{2}=30 \mathrm{fm}^{-2}$ (dash-dotted line). 


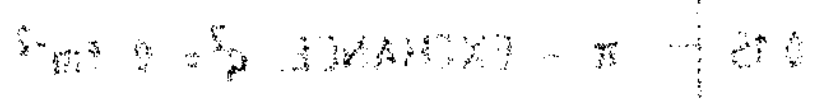

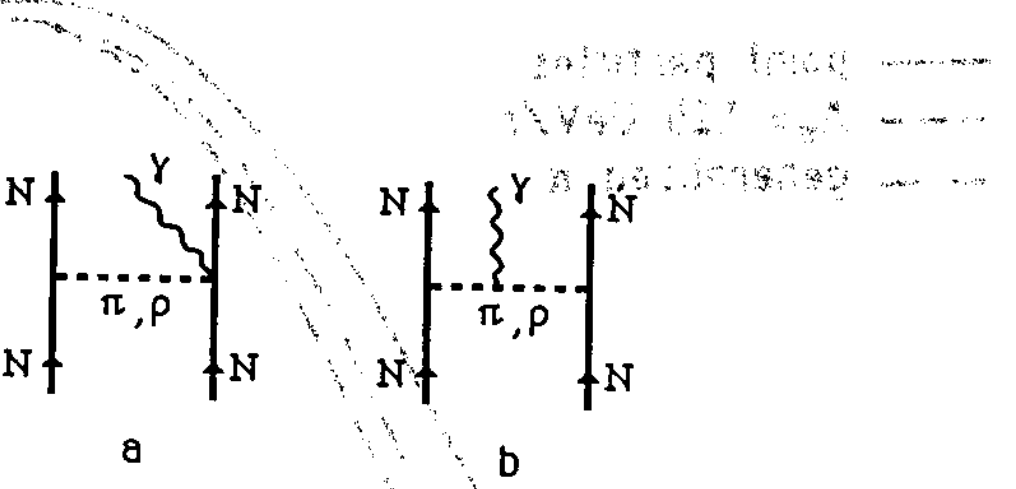

Figure 1

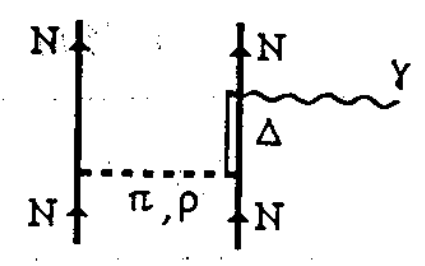

Figure 2 


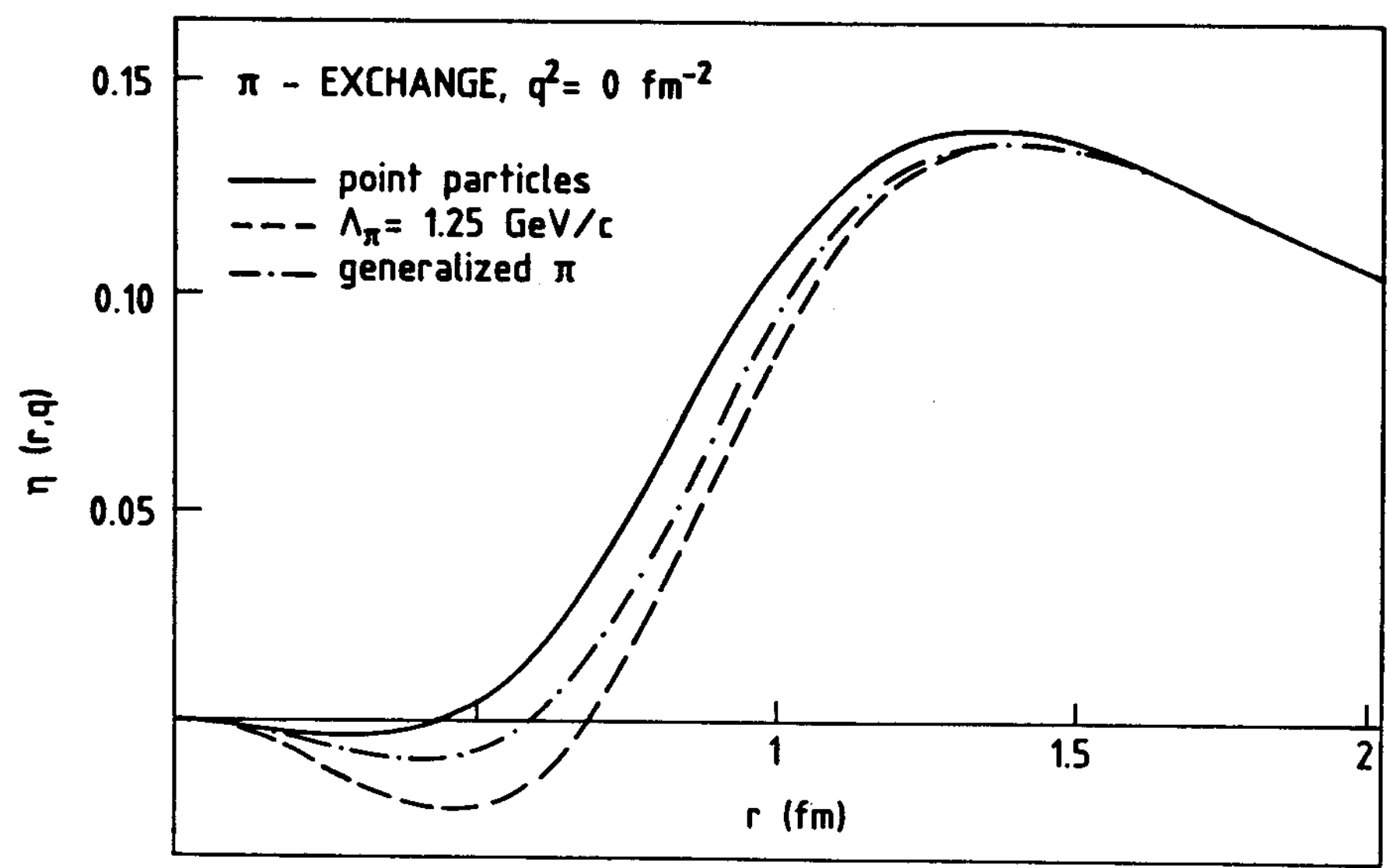

Figure 3

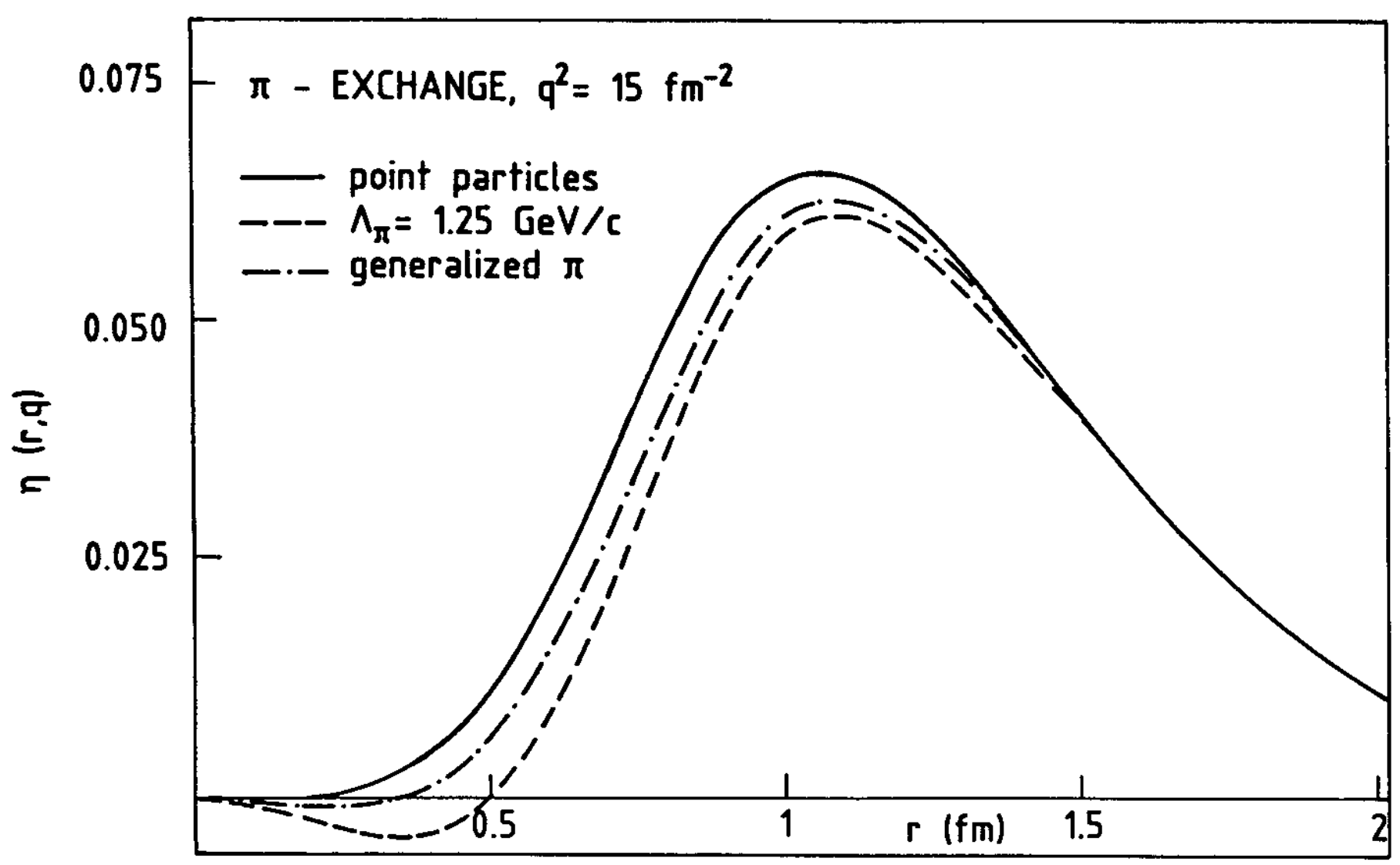

Figure 4 


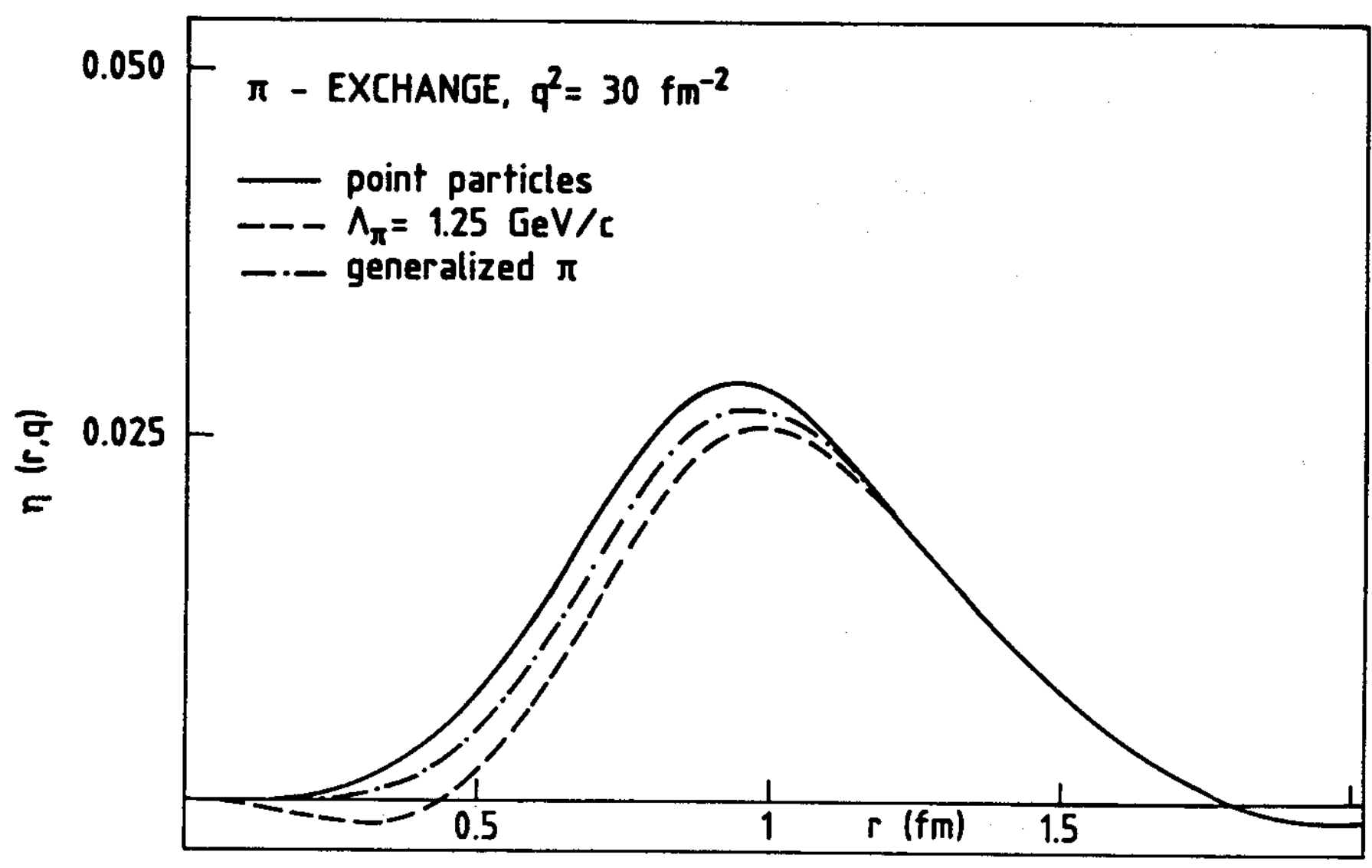

Figure 5

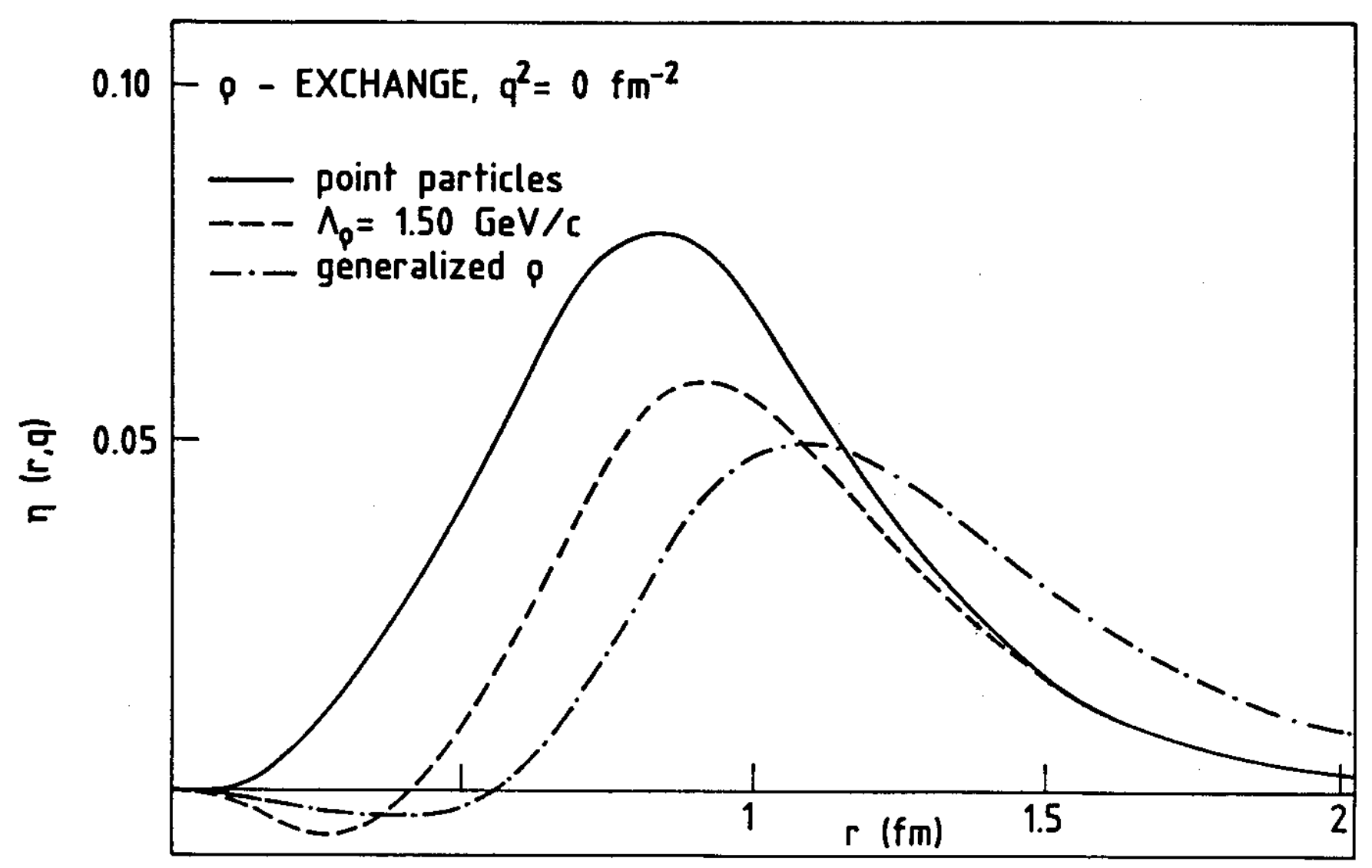

Figure 6 


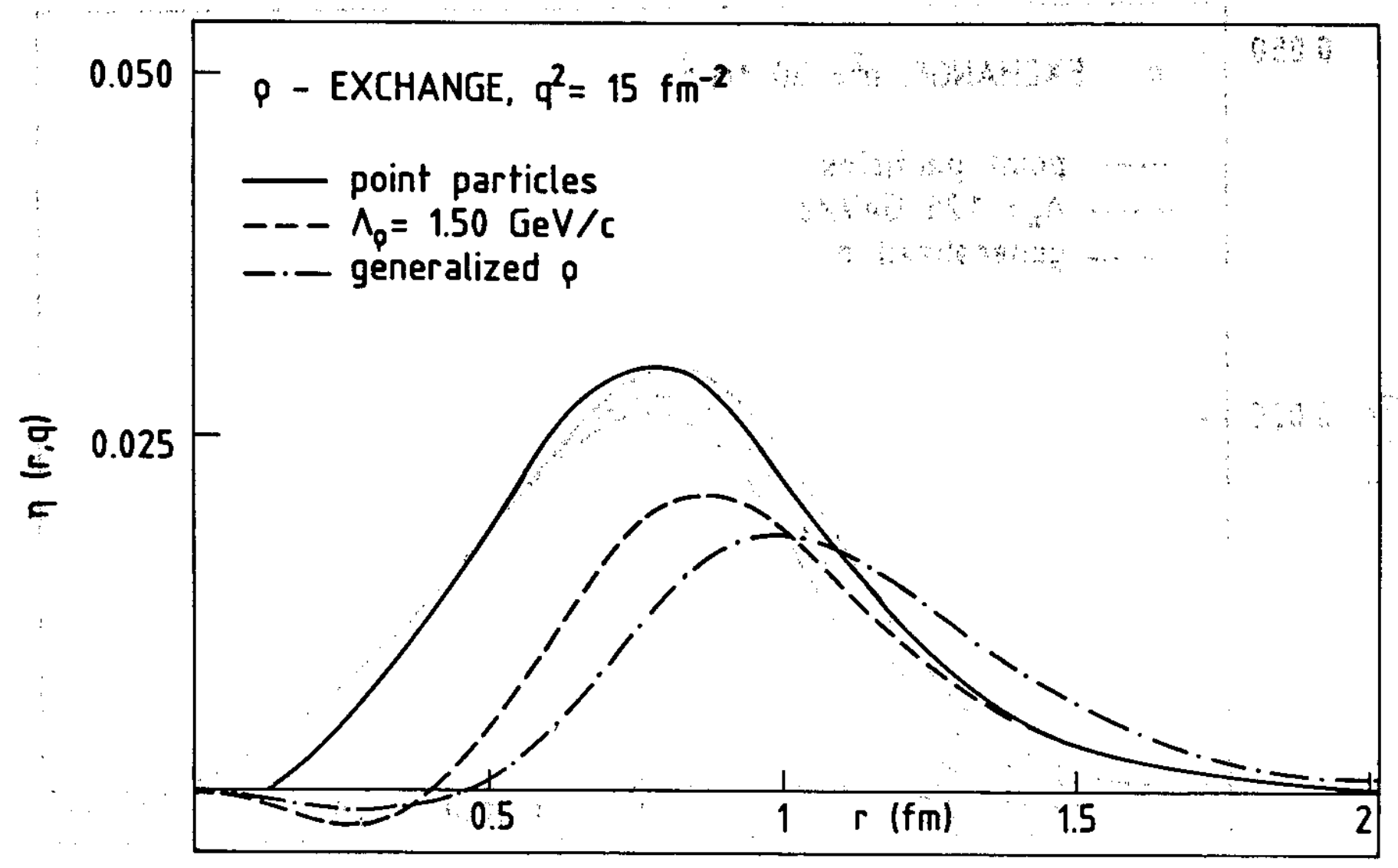

Figure 7

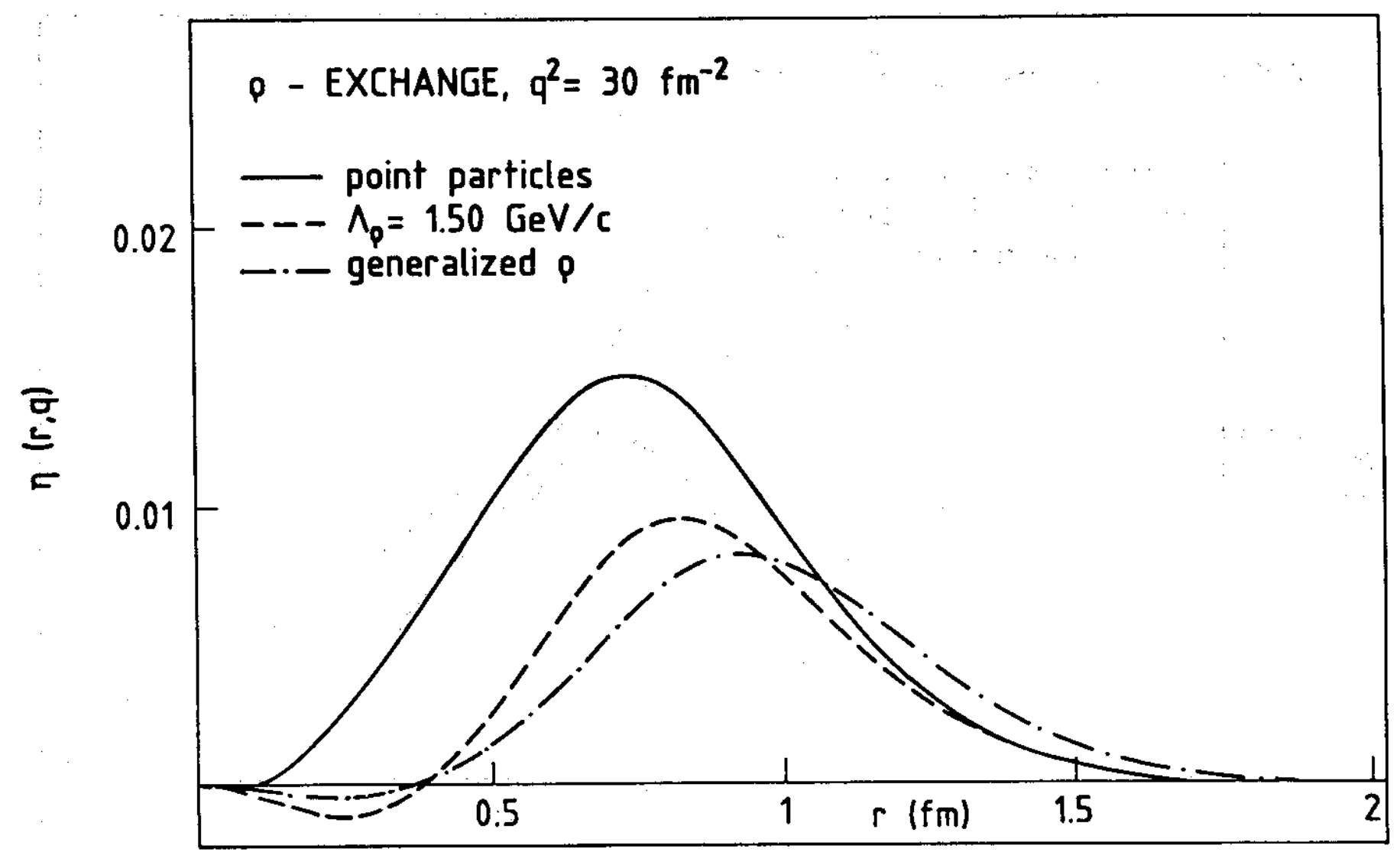

Figure 8 


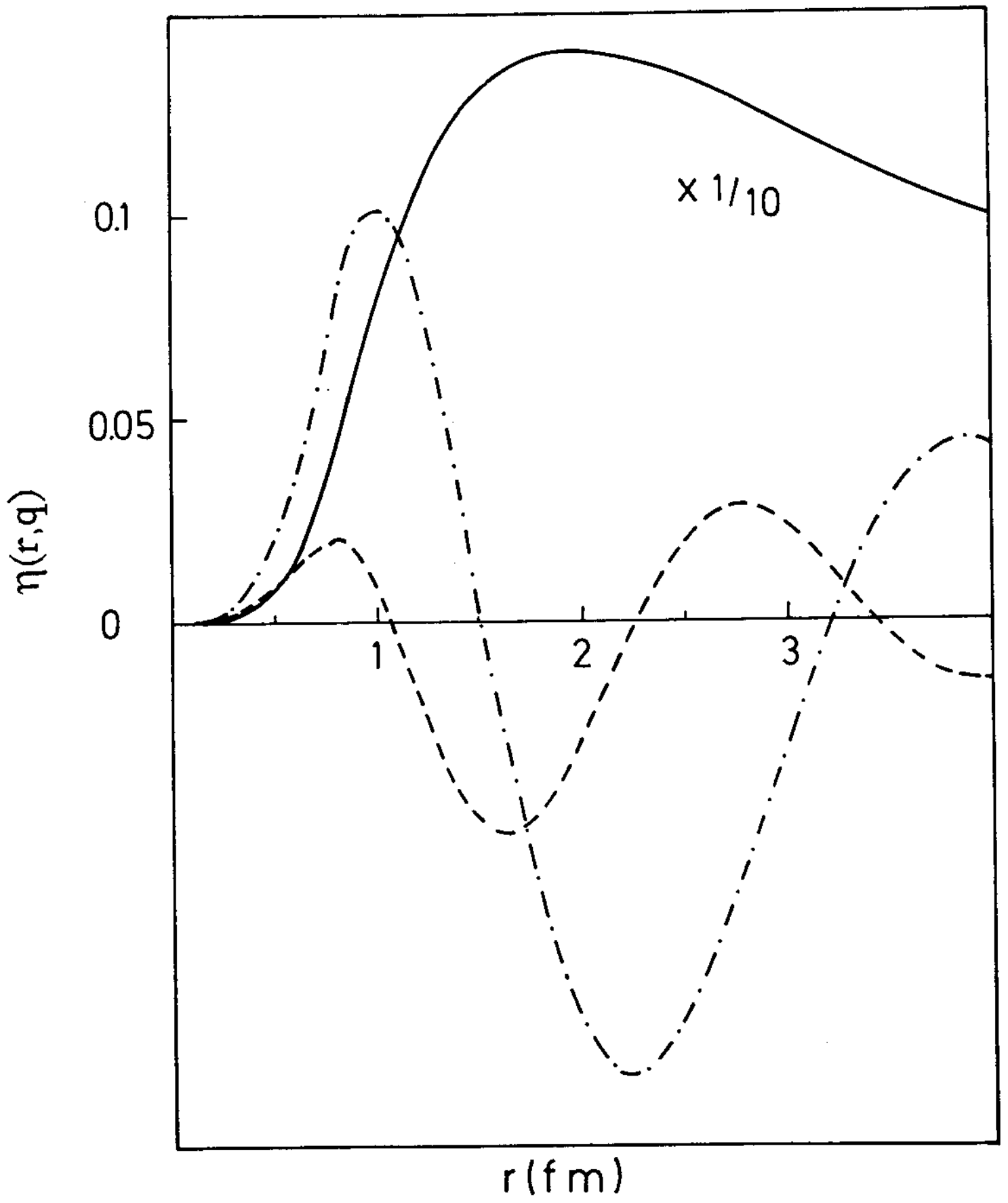

Figure 9 
